\title{
A hybrid model for multi-objective capacitated facility location network design problem
}

\author{
Mohammad Saeed JabalAmeli $^{\mathrm{a}^{*}}$ and Marzieh Mortezaei ${ }^{\mathrm{a}}$
}

${ }^{a}$ Department of Industrial Engineering, Iran University of Science \& Technology, Tehran, Iran

\begin{tabular}{|c|c|}
\hline ARTICLE I N F O & A B S T R A T T \\
\hline $\begin{array}{l}\text { Article history: } \\
\text { Received } 25 \text { January } 2011 \\
\text { Received in revised form } \\
\text { April, 5, } 2011 \\
\text { Accepted } 11 \text { April } 2011 \\
\text { Available online } \\
12 \text { April } 2011 \\
\text { Keywords: } \\
\text { Facility Location } \\
\text { Network design } \\
\text { Hybrid algorithm } \\
\text { Simulated annealing algorithm }\end{array}$ & $\begin{array}{l}\text { One of the primary concerns on many traditional capacitated facility location/network problems } \\
\text { is to consider transportation and setup facilities in one single objective function. This simple } \\
\text { assumption may lead to misleading solutions since the cost of transportation is normally } \\
\text { considered for a short period time and, obviously, the higher cost of setting up the facilities may } \\
\text { reduce the importance of the transportation cost/network. In this paper, we introduce } \\
\text { capacitated facility location/network design problem (CFLNDP) with two separate objective } \\
\text { functions in forms of multi-objective with limited capacity. The proposed model is solved using } \\
\text { a new hybrid algorithm where there are two stages. In the first stage, locations of facilities and } \\
\text { design of fundamental network are determined and in the second stage demands are allocated to } \\
\text { the facilities. The resulted multi-objective problem is solved using Lexicography method for a } \\
\text { well-known example from the literature with } 21 \text { node instances. We study the behaviour of the } \\
\text { resulted problem under different scenarios in order to gain insight into the behaviour of the } \\
\text { model in response to changes in key problem parameters. }\end{array}$ \\
\hline
\end{tabular}

(C) 2011 Growing Science Ltd. All rights reserved

\section{Introduction}

Simple observations prove that in some situations, it may be more cost effective to change the configuration of the underlying network instead of locating new facilities. In general, joint facility location/network design problems are useful for modelling a number of contexts in which tradeoffs between facility costs, network design and operating costs must be made. Such situations arise in the vast and diverse number of contexts such as, regional planning, distribution systems, airline networks, telecommunications, energy management, pipeline distribution systems, hub and spokes networks, LTL freight distribution system design and other areas.

Consider a set of nodes representing demand points as well as candidate facility locations, and a network of potential capacitated links. Each link can be either constructed or not at a given cost. Each node is associated with fixed charge for building a capacitated facility at that node and all demands must be served. The problem is to determine simultaneously the optimal locations of facilities and fundamental network, where the objective is to minimize the total construction, facility and link construction, and transportation costs.

* Corresponding author. Tel: +0982173220000

E-mail: jabal@iust.ac.ir (M. S. JabalAmeli) 
The capacitated facility location/network design problem (CFLNDP) was introduced by Melkote and Daskin (2001). The CFLNDP assumes that links may carry an infinite amount of demand which is often unrealistic since there are many applications from roads, telecommunication networks, pipelines where we can carry only a limited amount of network flow. In this paper, we introduce a variant of the CFLNDP in which links are capacitated. Depending on the intended application, a number of ways of modelling this extension exist such as applying some bounds on the link flows. During the past few years, there has been an increasing interest on CFLNDP problems. Magnanti et al. (1984) provided a brief overview of these problems. In general, researchers have found that capacitated network design problems are much more difficult to solve compared with uncapacitated counterparts (Magnanti, 1984).

\section{Related litreture}

Classical network location models locate facilities on a given network. However, the topology of the basic network may have a profound effect on the optimal facility locations. General network design issues are discussed in Friesz et al. (1998), Mitchel and Smith (2001), Solanki et al. (1998), and Yang (1998). The network design-location interface is discussed in Melkote and Daskin (2001) and Pardalos and Du (1998). Since the design of the underlying networks has a profound impact on the optimal facility location, some researchers tried to combine these two important fields (facility location and network design). Before the comprehensive study was done by Melkote and Daskin (2001), few attempts were made to explore the relationship of facility location and network topology. Application-specific models were also developed for some of the area mentioned above.

Berman et al.(1992) showed how the effectiveness (measured by transport costs) of existing facilities can be improved by making certain changes to the configuration of the fundamental network. Peeters and Thomas (1995) investigated the impact of different network topologies on the optimal solutions to the p-median problem, finding, not surprisingly, that the effect is "significant". Bhadury et al.(2003) studied a facility layout plannign which is located at a certain node of a network and found the more economical spanning tree based on 1-median model. Drenzer and Weosowlky(1998) presented a heuristic for finding a configuration of one-way and two-way streets and the location of a single facility in a network to minimize the total transportation costs. Drenzer and Weosowlky (2003) also examined some meta heuristic algorithms to solve this problem and compared the effectiveness of algorithms.

Another related research erea deals with location-routing problems. These problems simultaneously consider the location of facilities and delivery/cllection routes or paths. Laporte (1998) provided overviews of these problems. Another related line of work is the hub location problems. Hub location models can also be considered as joint location / network design problems in the sense that they simultaneously address where to locate the hubs and how to design the hub-level network and the access-level network. Campbell (1994) and Klincewicz (1998) provided a comprehensive overview of these models. Daskin (1993) introduced the incapacitated facility location/network design problem (UFLNDP). Melkote and Daskin (2001) solved instances of the UFLNDP with up to 40 nodes and 160 candidate links and identify a number of cases of the problem which could be solved in a reasonable amount of time. They also expanded the model for the capacitated facilities (2001). There are many works on FLNDP where the focus is on developing efficient solution algorithms consist of heuristic and meta-heuristic algorithms. In this paper, we introduce capacitated facility location/network design problem (CFLNDP) with two separate objective functions in forms of multiobjective with limited capacity. The proposed model is solved using a new hybrid algorithm where there are two stages. In the first stage, locations of facilities and design of fundamental network are determined and in the second stage, demands are allocated to the facilities.

The rest of this paper is organized as follows: In the next section we give a bi-objective mixed integer programming formulation of our model. In section 4, the proposed hybrid algorithm to solve the problem is presented. In order to gain insight on the behaviour of the model, some sensitivity analysis is performed on a problem instance in section 5. We draw conclusions in Section 6. 


\section{Problem formulation}

Before formulating the CFLCNDP, we make the following assumptions concerning the fundamental network based on Melkote and Daskin (2001):

1. Each node represents a demand point,

2. Facilities may only be located at the nodes of the network,

3. Only one facility may be located per node,

4. The network is a customer-to-server system

5. All travel costs are symmetric.

6. All networks link are directed.

To formulate the problem we define the following notation:

Inputs

$\mathcal{N}$ set of nodes in the network

$\mathcal{L} \quad$ set of undirected candidate links in the network

$d_{i} \quad$ demand at node $\mathrm{i}$

$M \quad \sum_{\mathrm{i} \in \mathrm{N}} \mathrm{d}_{\mathrm{i}}$ (total network demand)

$t_{i j} \quad$ travel cost per unit flow on link $(\mathrm{i}, \mathrm{j})$

$f_{i} \quad$ cost of constructing a facility at node $\mathrm{i}$

$K i \quad$ capacity of facility constructed at node $\mathrm{i}$

$c_{i j} \quad$ cost of constructing link $(\mathrm{i}, \mathrm{j})$

$U_{i j} \quad$ maximum amount of flow can be carried on link $(\mathrm{i}, \mathrm{j})$

$t_{i j}^{0} \quad$ travel cost per unit flow on link $(\mathrm{i}, \mathrm{j})$

The demand originating at node $\mathrm{k}$ is defined a "commodity" $\mathrm{k}$. Then the size of all commodities is scaled to unity and a commodity-specific travel cost is defined as:

$t_{i j}^{k} \quad$ travel cost of commodity $\mathrm{k}$ on link $(\mathrm{i}, \mathrm{j})=\mathrm{d}_{\mathrm{k}} \mathrm{t}_{\mathrm{ij}}^{0}$

Decision variables

$Z_{i}= \begin{cases}1 & \text { if a facility is located at node } \mathrm{i} ; \\ 0 & \text { if not; }\end{cases}$

$X_{i j}= \begin{cases}1 & \text { if link }(\mathrm{i}, \mathrm{j}) \text { is constructed, where } \mathrm{i}<j ; \\ 0 & \text { if not; }\end{cases}$

$Y_{i j}^{k}=$ the fraction of commodity $\mathrm{k}$ that flows on link $(\mathrm{i}, \mathrm{j})$,

$W_{i}^{k}=$ the fraction of commodity $\mathrm{k}$ served by a facility at node $\mathrm{i}$

We assume all inputs are nonnegative. Then our formulation is:

(CFLCNDP)

$\min \sum_{(i, j) \in L} \sum_{k \in N} t_{i j}^{k} Y_{i j}^{k}$

$\min \sum_{i} f_{i} Z_{i}++\sum_{(i, j)} C_{i j} X_{i j}$

subject to

$Z_{i}+\sum_{j \in N} X_{i j} \geq 1$

$i \in N$

$\sum_{j \in N} Y_{j i}^{k}=\sum_{j \in N} Y_{i j}^{k}+W_{i}^{k}$

$i, k \in N: i \neq k$

$W_{i}^{i}+\sum_{j \in N} Y_{i j}^{i}=1$,

$i \in N$ 
$\sum_{i \in N: i \neq k} W_{i}^{k}=1$

$$
\sum_{k \in N} d_{k}\left(Y_{j i}^{k}+Y_{i j}^{k}\right) \leq U_{i j}
$$$$
(i, j) \in L, k \in N
$$

$\sum_{k \in N} d_{k} W_{i}^{k} \leq K_{i} Z_{i}$

$i \in N$

$Y_{i j}^{k} \leq X_{i j}$,

$X_{i j}+X_{j i} \leq 1$,

$(i, j) \in L, k \in N$

$(i, j) \in L$

$Z_{i} \in\{0,1\}, X_{i j} \in\{0,1\}(i, j) \in L$,

$W_{i}^{k} \geq 0,0 \leq Y_{i j}^{k} \leq 1$

The first objective function in Eq. (1) minimizes the transportation cost and the second objective function given in Eq. (2) minimizes the sum of facility location and link construction costs -in other words minimizing the amount of investment. Eq. (3) means that the demand at node $i$ is served by a facility at node $i$ and/or using one or more outbound links (leading to different facilities). Eq. (4) states that each demand entering node $i$ is either shipped out or served at node $i$, and Eq. (5) states that demand at node $i$ must be served either by a facility at node $i$ or by another facility. Eq. (6) also states that all demands must be served. Eq. (7) guarantees that total carried demands on a link cannot exceed link capacities. Similarly Eq. (8) ensures that demand is only served at located facilities and that facility capacities are not exceeded. Eq. (9) states that flow is permitted only on constructed links. Eq. (10) prevents oppositely directed flows on a link and Eqs. (11) state the standard nonnegativity and binary variables. If we set $U_{i j}=\infty$ for all links (i.e., make all uncapacitated links) the problem is converted into CFLNDP. Since Melkote and Daskin (2001) already explained that the CFLNDP is NP-hard the CFLCNDP is also NP-hard.

\section{The hybrid algorithm for CFLCND problem}

Since the CFLCNDP is NP-hard we introduce a hybrid algorithm which solves the problem in 2 stages. In the first stage, locations of facilities and design of fundamental network are determined and in the second stage, demands are allocated to the facilities.

\subsection{First stage}

The first stage consists of two phases; phase I generates an initial solution and the phase II improves the present solution using simulated annealing (SA) algorithm and these two stages are described next.

\subsubsection{Phase I: Initial solution generation}

The quality of the initial solution plays an important role for the quality of the final solution obtained by the SA algorithm. In this paper, we use the following heuristic algorithm to generate relatively high quality initial solution, which satisfies capacity constraints.

The heuristic algorithm includes the following steps:

- Step 1: Estimate the optimal number of required facilities (c),

- Step 2: Locate the facilities and allocate the demands to them, $a$ : small scale problems (complete enumeration),

$b$ : large scale problems (using Fuzzy Clustering Method),

- Step 3: Find the shortest paths between located facilities and the demand nodes allocated to each one,

- Step 4: Investigate whether the capacity constraints related to facilities and links are satisfied, otherwise make required changes, 


\subsubsection{Phase II: Improvement of the initial solution}

Once the initial solution is generated, we can improve its quality using the SA algorithm. The SA algorithm is described in the next section.

\subsubsection{Simulated annealing}

SA is a generic probabilistic meta-heuristic for the global optimization problem of applied mathematics, namely locating a good approximation to the global minimum of a given function in a large space. It is often used when the search procedure space is discrete. SA is a stochastic search procedure based on decreasing temperatures consciously. SA was introduced by Metropolic et al. (1953) and popularized by Kirkpatrick et al. (1983). Unlike many local search algorithms, all moves which lead to worse solutions are accepted in SA based on a probability which is an initial solution and the beginning temperature are chosen. SA evaluates the value of the cost function according to the current solution. Once the best solution from the neighbourhood of the current solution is obtained, a comparison will be made between the new value of the cost function based on the solution and the current cost function. New cost function value will be accepted if its value is less than the current value, otherwise the new value would be accepted only when the Metropolis's criterion which is based on Boltzman's probability is met. The cost function values of the current and the newly generated solutions $(\Delta \mathrm{E})$ are equal to or larger positive value. Therefore, a random number $\delta$ in $[0,1]$ is generated based on a uniform distribution where we have $\delta \leq e^{\frac{-\Delta E}{T}}$. If Eq. (4) is met, the newly generated solution is accepted as the current solution. The SA algorithm is terminated when it reaches to a certain number of iterations or a number of iterations without improving the objective function value are executed. The temperature is lowered based on the temperature updating rule, and according to this rule a geometric scale factor is often used to be multiplied by current temperature. SA results are highly sensitive to the number of iterations at each temperatures and the speed of reducing temperature.

The SA procedure is as follows:

Input: Basic solution ( $\pi$ current $=$ random solution or the best known solution $(\pi$ current $=\pi 0)$ ); Sbest=fitness of $\pi$ current; SA parameters and mechanisms such as the cooling rate, starting temperature, neighbourhood generation mechanism, maximum number of iterations, Scale Factor, etc.

Output: Best solution found ( $\left.\pi_{\text {best }}\right)$

\section{Procedure:}

$\mathrm{T}=\mathrm{T}_{0}$

While (Termination criteria not met)

For $\mathrm{i}=1$ : MaxIter

$\mathrm{T}=\mathrm{T}_{0} *$ ScaleFactor

Generate neighbour solutions for $\pi_{\text {current }}$ and find the solution with the best objective function value $\left(\Omega_{\text {NBest }}\right)$.

If $\left(\Omega_{N \text { Best }} \leq \Omega_{\text {best }}\right)$

Else

$\pi_{\text {best }}=\pi_{\text {NBest }}$ and $\Omega_{\text {best }}=\Omega_{\text {NBest }}$

$\Delta E=\Omega_{\text {NBest }}-\Omega_{\text {best }}$

$\mathrm{RAND}=$ a random number between 0 and 1 .

If $R A N D \leq e^{(-\Delta E / T)}$

$\pi_{\text {best }}=\pi_{\text {NBest }}$ and $\Omega_{\text {best }}=\Omega_{\text {NBest }}$

End if

End if

End

End

Return $\pi_{\text {best }}$ 


\subsubsection{Solution Representation}

The proposed solution consists of two- parts and it can be explained as follows:

Given $N$ nodes and $L$ potential links which can be drawn in two directions, a chromosome will have $N+2 L$ elements, in which $N$ cells belong to the first part, and $2 L$ cells belong to the second part. The first part of the vector shows the location of facilities and the second part shows the selected links of the fundamental networks.

For instance, a Daskin problem with 6 nodes and its solution representation are presented in Fig. 1. In this case, there are 6 demand nodes and 4 potential links which can be considered from each side.

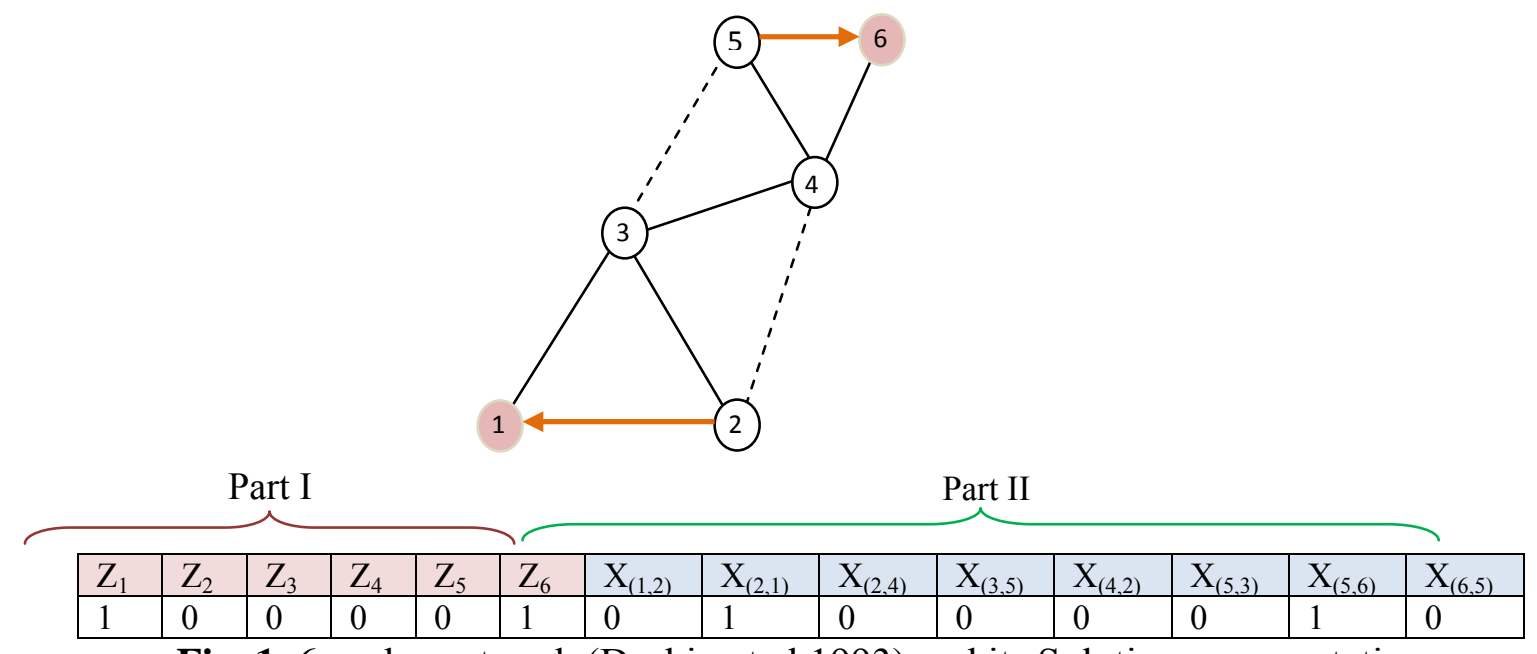

Fig. 1. 6-nodes network (Daskin et al.1993) and its Solution representation

\subsubsection{Neighbourhood Search Structure}

The efficiency of any meta-heuristic algorithm which uses neighbourhood structures to search the solution space severely depends on how the neighbourhood structures used in the algorithm are defined. The number of neighbourhood structure and the order according to which these structures are used in the local search procedure affect both the quality of the solutions and the amount of time needed to reach such solutions. We propose the following five neighbourhood structures in our study to search the solution space in the SA algorithm. The first two neighbourhood structures affect the facility location related part to the solutions while the last three neighbourhood structures address the network design related part of the solutions. We illustrate these five NSS on the 6-nodes network.

N1(x): In this Neighbourhood Search Structure (NSS) the 2-opt movement is taken. 2 elements are selected randomly and their values are exchanged. By the 2-opt movement the number of facilities is not changed and just one of the facilities is opened and another facility is closed.

\begin{tabular}{|l|l|l|l|l|l|l|l|l|l|l|l|l|l|}
\hline 1 & 0 & 0 & 0 & 0 & 1 & 1 & 0 & 0 & 0 & 0 & 0 & 0 & 1 \\
\hline \multicolumn{10}{|c|}{} \\
\hline 0 & 0 & 0 & 1 & 0 & 1 & 1 & 0 & 0 & 0 & 0 & 0 & 0 & 1 \\
\hline
\end{tabular}

Fig. 2. Neighborhood 1

N2(x): In this NSS, one element is selected randomly. If its value is 0 it will be changed into 1 and vice versa. By this movement it would be possible that the number of facilities is changed, but this change is in a limited domain. That is, the number of facilities can decrease or increase one unit.

\begin{tabular}{|l|l|l|l|l|l|l|l|l|l|l|l|l|l|}
\hline 1 & 0 & 0 & 0 & 0 & 1 & 1 & 0 & 0 & 0 & 0 & 0 & 0 & 1 \\
\hline \multicolumn{10}{|c|}{} \\
\hline 1 & 0 & 1 & 0 & 0 & 1 & 1 & 0 & 0 & 0 & 0 & 0 & 0 & 1 \\
\hline
\end{tabular}

Fig. 3. Neighborhood 2 
N3(x): In this NSS the r-opt movement is taken and $r$ is selected randomly between 0 and $2 L$. Then $r$ cells from the last part of chromosome will be chosen and their values exchanged. The $r$-opt movement do not make any changes in the number of links.

\begin{tabular}{|c|c|c|c|c|c|c|c|c|c|c|c|c|c|}
\hline 1 & 0 & 0 & 0 & 0 & 1 & 1 & 0 & 0 & 0 & 0 & 0 & 0 & 1 \\
\hline \multicolumn{10}{|c|}{} \\
\hline 1 & 0 & 0 & 0 & 0 & 1 & 1 & 0 & 1 & 0 & 0 & 0 & 0 & 0 \\
\hline
\end{tabular}

Fig. 4. Neighborhood 3

$N 4(x)$ : In this NSS the $r$-opt movement is taken, $r$ is selected randomly between 0 and $2 L$ and $r$ cells from the last part of chromosome are chosen. If their values are 0 they will be changed into 1 and vice versa. By this movement the number of links might change.

\begin{tabular}{|c|c|c|c|c|c|c|c|c|c|c|c|c|c|}
\hline 1 & 0 & 0 & 0 & 0 & 1 & 1 & 0 & 0 & 0 & 0 & 0 & 0 & 1 \\
\hline \multicolumn{10}{|c|}{} \\
\hline 1 & 0 & 0 & 0 & 0 & 1 & 1 & 1 & 0 & 1 & 0 & 0 & 1 & 1 \\
\hline
\end{tabular}

Fig. 5. Neighborhood 4

N5(x): In the CFLCNDP movement on the links is allowed just in one direction. Eq. (9) shows this matter. After generating a solution and before calculating cost of this solution, we check the solution in Eq. (9). If this equation is not satisfied, one of the symmetry elements is chosen randomly and the value changed to 0 . Since this choice is made randomly, N5(x) is defined to resolve this problem. In this NSS one of the cells is selected and its value is exchanged by the symmetrical element.

\begin{tabular}{|l|l|l|l|l|l|l|l|l|l|l|l|l|l|}
\hline 1 & 0 & 0 & 0 & 0 & 1 & 1 & 0 & 0 & 0 & 0 & 0 & 0 & 1 \\
\hline \multicolumn{10}{|c|}{} \\
\hline 1 & 0 & 0 & 0 & 0 & 1 & 1 & 0 & 0 & 0 & 0 & 0 & 1 & 0 \\
\hline
\end{tabular}

Fig. 6. Neighborhood 5

\subsection{Second stage: Computing optimal allocations}

After locating the facilities and designing the fundamental network, to minimize the transportation cost we should determine the optimal allocations. In other words, we must solve the linear programming, which is a linear transportation problem virtually. Although simplex algorithm can also be used to solve it, the network simplex algorithm can obtain the optimal solution much easier and faster. So we use the network simplex algorithm to solve the allocation problem. The network simplex algorithm is a well-known simplex algorithm. For detailed expositions, the interested reader may consult Bazaraa et al. (1990).

\section{Computational study}

In this section, we evaluate the performance of hybrid algorithm to solve the CFLCNDP. The proposed solution algorithm is coded in MATLAB R2008b and it was run on a PC with an Intel Core 2 Duo CPU (2.0 GHz) and 2 GB memory. In addition, Cplex MIP solver has been used for computing the optimal allocations. In order to verify the proposed approach, 12 test network are randomly generated and results obtained by applying the hybrid algorithm on these problem sets are compared to the solutions provided by Cplex MIP solver.

\section{1 test networks}

We use the same procedure utilized by Daskin et al. (2000) to generate test networks for the UFLNDP. These networks vary in size from 10 to 100 nodes and $2 \mathrm{~N}$ candidate links. The procedure is as follow:

First, the desired number of nodes is generated on a $100 * 100$ grid, with their $\mathrm{x}$ - and $\mathrm{y}$-coordinates randomly sampled from a Uniform $(0 ; 100)$ distribution. Then the desired number of candidate links is randomly selected, with a bias toward shorter links to emulate transportation networks. We then 
calculate the Euclidean length of each link and round it to the nearest integer. The nodal demands are sampled from a Uniform $(10 ; 40)$ distribution, the fixed facility charges are sampled from a Uniform $(50000 ; 150000)$ distribution and both are rounded to the nearest integer.

\subsection{Parameter setting}

Initial temperature and structure of making changes in the temperature are the most important parameters in the SA algorithm. In the proposed algorithm the temperature changes with the structure of $T_{n+1}=\alpha \times T_{n}$ and $\alpha$ is assumed to be 0.95 . In order to determine the initial temperature, we use the procedure introduced by Crama and Schyns (2003). In this method the goal is to gain the equal acceptance probability (here $\chi_{0}=0.8$ ) through the $\mathrm{L}$ first stages of the algorithm. Then average increment in the objective function is calculated and denoted by $\Delta$. Ultimately the initial temperature is computed as follows:

$$
T_{0}=\frac{\Delta}{\ln \chi_{0}}
$$

The other issue is to determine the chances of applying changes in NSS to find the optimum solution by the algorithm. By the use of tuning the best proportions for implementation of each change on the present solution is obtained as follows:

Table 1

Proportion for the implementation of each change

\begin{tabular}{ccccc}
\hline$N_{1}(x)$ & $N_{2}(x)$ & $N_{3}(x)$ & $N_{4}(x)$ & $N_{5}(x)$ \\
\hline 0.35 & 0.05 & 0.25 & 0.25 & 0.1 \\
\hline
\end{tabular}

\subsection{Evaluation of the results}

In summary, we solved 12 test networks to test the performance of the proposed hybrid algorithm. In order to evaluate the efficiency of our hybrid algorithm, we have compared the results obtained by the hybrid algorithm by those obtained by the exact solution procedure. In Table 2, the first column represents the number of nodes, the second, the third and the forth columns respectively represent the minimum, mean and maximum objective function values obtained by hybrid algorithm via 10 times solving each instance problem.

Columns five and six represent the objective function values provided by exact solution procedure (we used the Cplex MIP solver for solving the problem) for each problem instance and the relative gap (in percentage) between the objective function values of the hybrid algorithm and those of the exact solution, respectively. According to the results reported in Table 2, the hybrid algorithm provides high quality solutions with the objective function values not more than $9 \%$ (in average) of the objective values of the solutions obtained by the exact solution procedure.

Table 2

Computational experience for the set of randomly generated test problems

\begin{tabular}{|c|c|c|c|c|c|}
\hline \multirow{2}{*}{ ID } & \multicolumn{3}{|c|}{ Solution obtained by the hybrid algorithm } & \multirow{2}{*}{$\begin{array}{l}\text { Optimal } \\
\text { solution }\end{array}$} & \multirow{2}{*}{$\%$ Gap } \\
\hline & Min & Mean & Max & & \\
\hline 6-nodes & 126660 & 129640 & 132993 & 126660 & $\% 0.00$ \\
\hline 10-nodes & 141770 & 146688 & 150276 & 141770 & $\% 0.00$ \\
\hline 15-nodes & 147734 & 152223 & 158075 & 147690 & $\% 0.03$ \\
\hline 21-nodes & 338974 & 351149 & 366092 & 338940 & $\% 0.01$ \\
\hline 30-nodes & 372834 & 385014 & 406389 & 372834 & $\% 0.00$ \\
\hline 40-nodes & 411347 & 429066 & 452482 & 410117 & $\% 0.3$ \\
\hline 50 -nodes & 557818 & 581207 & 619178 & 550117 & $\% 1.4$ \\
\hline 60 -nodes & 693899 & 738357 & 777167 & 685129 & $\% 1.28$ \\
\hline 70-nodes & 782116 & 864134 & 907255 & 753629 & $\% 3.78$ \\
\hline 80-nodes & 944161 & 1045629 & 1099948 & 891643 & $\% 5.89$ \\
\hline 90-nodes & 1098576 & 1226114 & 1285334 & 1028629 & $\% 6.8$ \\
\hline 100-nodes & 1270330 & 1341023 & 1524396 & 1166618 & $\% 8.89$ \\
\hline
\end{tabular}




\section{Sensitivity analysis}

In this section we perform some sensitivity analysis on a particular test problem with the intention of providing further insight into the behavior of the model. This problem, shown in Fig. 7, is a widely used test network (Daskin et al., 2005), We have scaled the travel costs up by a factor of 20. The travel cost $t_{i j}$ of each candidate link may be interpreted as its length. We assume that all travel costs are symmetric so that $t_{i j}=t_{j i}$ for all $(i ; j)$. Also, all distances on this network satisfy the triangle inequality.

All links to be constructed are assumed to have the same capacity, so all $U_{i j}$ are replaced with simply $U$. This parameter is varied from 10 to 100 in increments of 10 . Note that if we consider 0 as a starting value of capacity, we will be forced to construct facility at all nodes to be able to serve the total demands of the network, therefore 10 is assumed to be starting value. Here for simplicity, capacity of all facilities is assumed to be equal to 300 .

Figs. 8-14 contain plots of the model's output versus the capacity $U$, with the unit link construction cost $u$ fixed at 20 in all cases. (The problem was solved to optimality in all cases.)

LEGEND

$\$ 994$

(41)

50

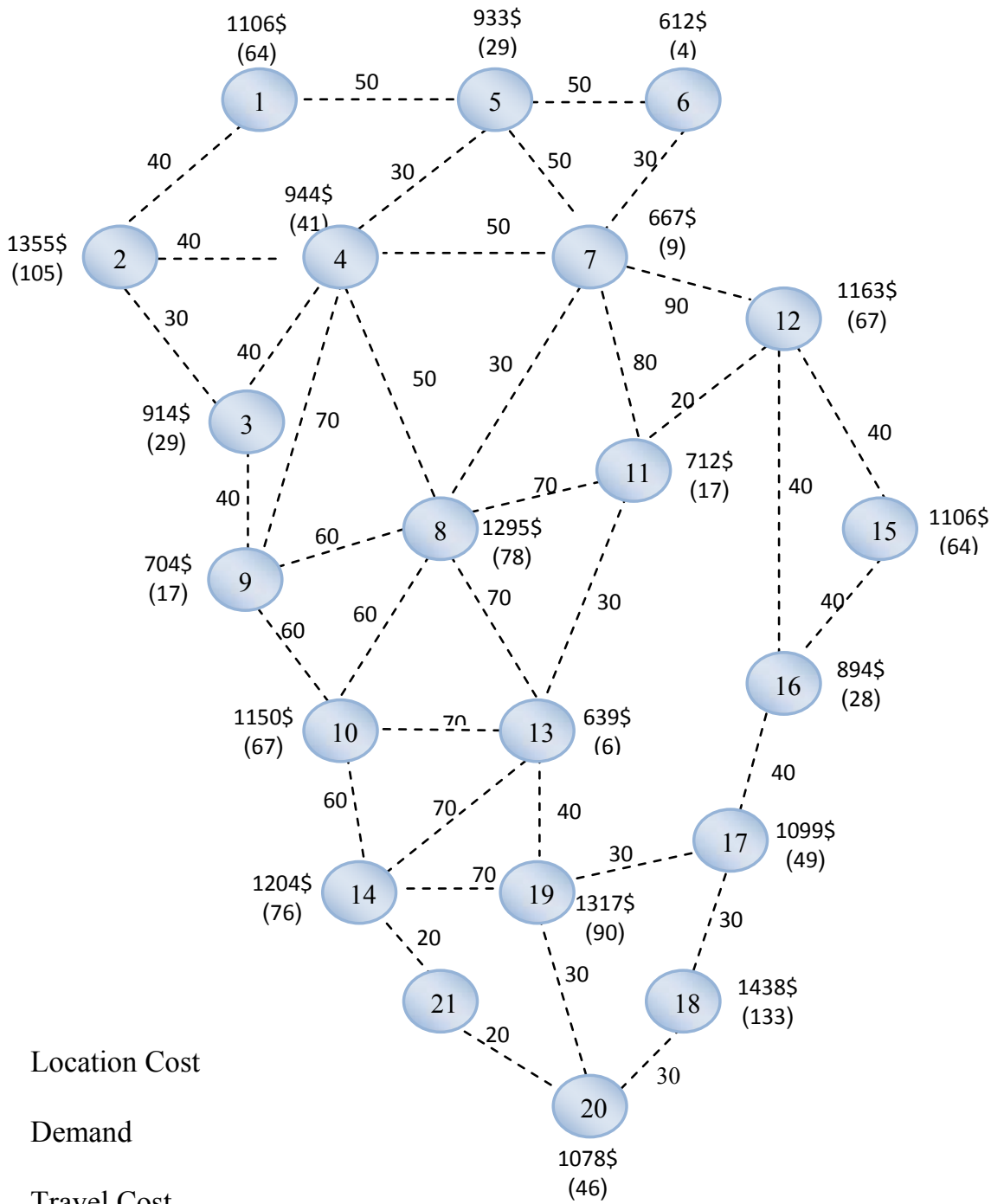

Candidate Link

When the capacity increases, we expect the number of facilities and accordingly facility investment decreases. This is evident from Fig. 9 and Fig. 10, which respectively plot the number of facilities and facility investment as a function of capacity. This diminishing in the number of facilities will 
cause increment in the number of multiply sourced demands, the number of links, link investment and consequently transportation costs. Figs. 11-13, depict this phenomenon.

We can see the behavior of the individual components of the objective function in Figs. 10, 12, and 13, which respectively display the facility and link investment and transportation costs versus the capacity. Finally, the most significant goal which is the reduction on total cost decrease is accomplished. This is evident from Fig. 14, which plots the total cost as a function of capacity. Fig. 8 shows that, as expected, the minimum budget is required for constructing facilities and links decreases monotonically as capacity is increased, since more link capacity allows us to invest in fewer facilities and use the constructed links to transmit the same demands to the facility nodes to be served.



Fig.8. Minimum budget is required vs. capacity.

In Fig. 9, as expected, we see the number of facilities located also decreases monotonically as capacity is increased. It is noticeable that when $U$ is close to $U_{\min }$ many demands must be served by the facilities located at the same nodes, since the demand is much more than the link capacity. This will result in a high level of facility investment. Once $U$ is slowly increased; we see a large decrease in the number of facilities that should be located. This allows us to substantially reduce facility investment.

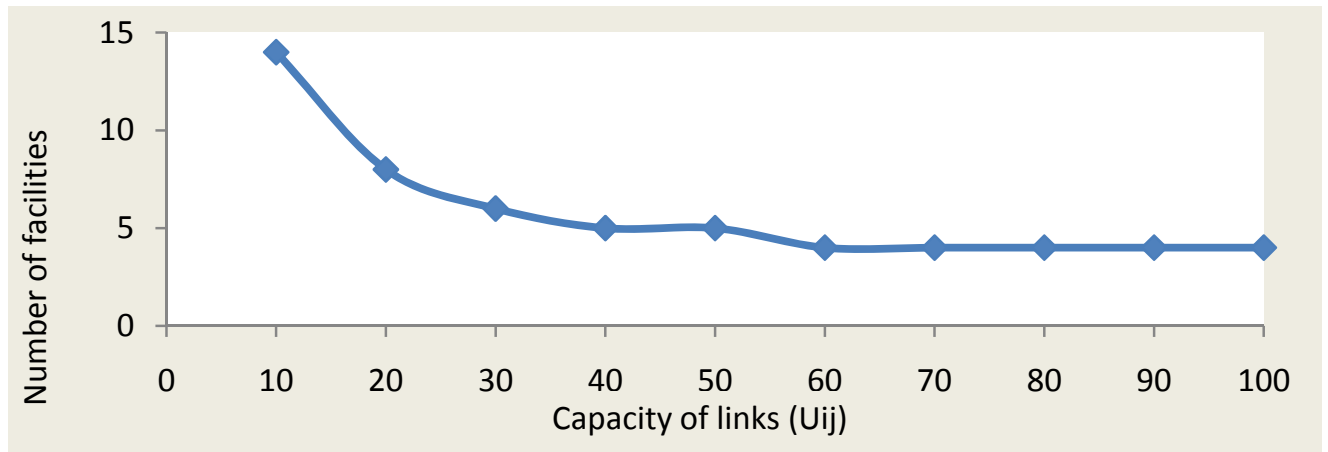

Fig.9. Number of facilities vs. capacity

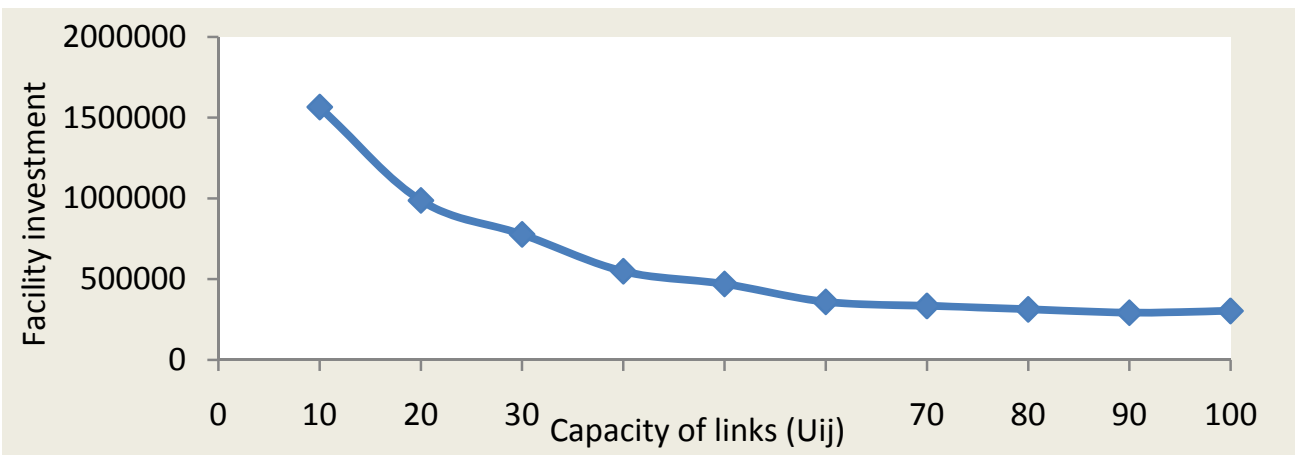

Fig.10. Facility investment vs. capacity 
Fig. 10 shows that facility investment monotonically decreases as capacity is increased, as expected, since more capacity allows us to invest in fewer facilities to serve the same demands. Fig. 11 shows that, capacity increment make it possible to carry more demands on the links. Therefore, we expect the number of links decrease versus the capacity increase. However, as shown in Figs. 11 and 12, the number of links and accordingly link investment increase. The explanation for this behavior becomes clearer if we compare the facility and link construction costs. Since facility construction cost is very large compared with link construction costs, we prefer to construct more links and transmit the demand to the facility nodes. The model significantly reduces investment in facilities instead, trading off higher link expenditures to serve the demands that were served at the former facility nodes. This is evident when we observe that for every increase in link investment in Fig. 12, there is a substantial corresponding drop in facility investment and/or the number of facilities located.

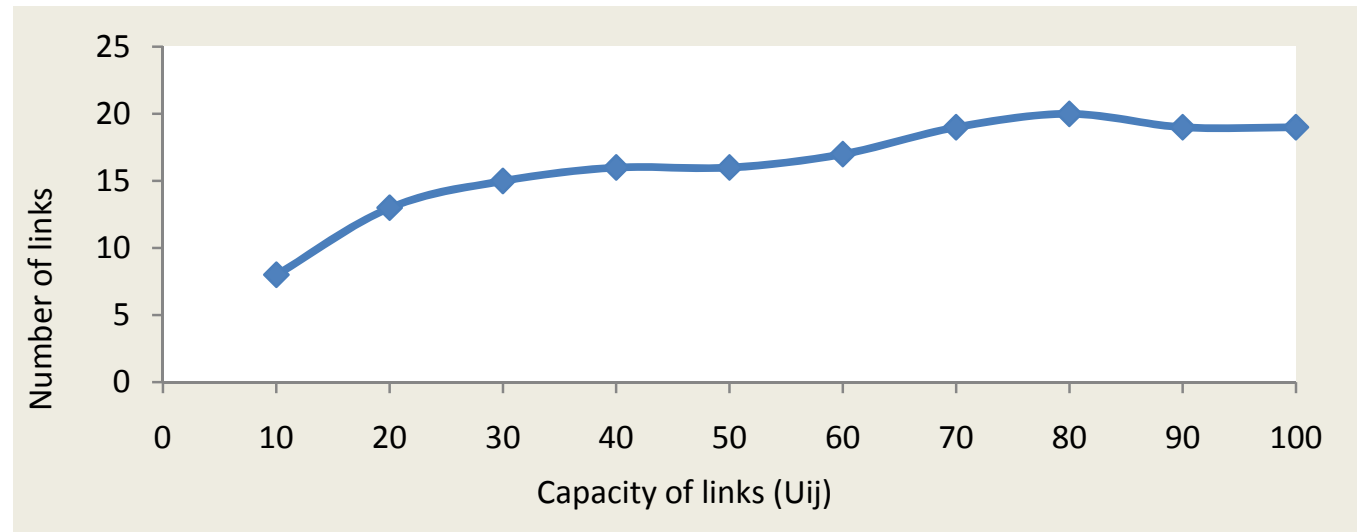

Fig. 11. Number of Links vs. capacity

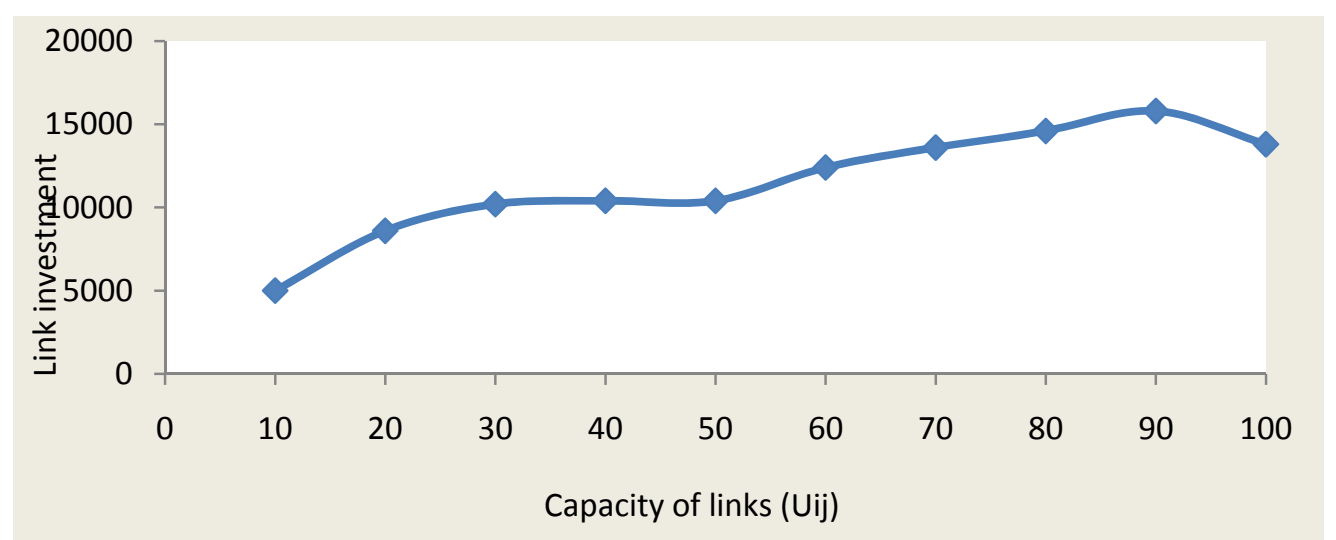

Fig. 12. Link investment vs. capacity

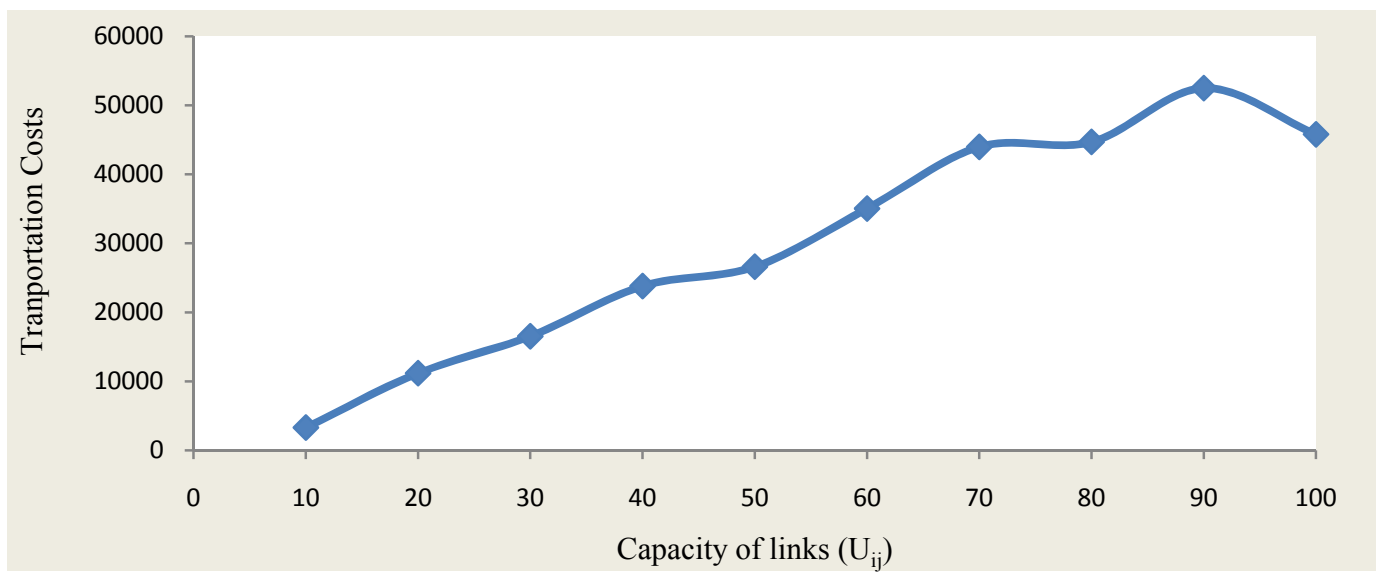

Fig. 13. Transportation costs vs. capacity 
Fig. 13 contains the transportation costs plotted as a function of link capacity. The plot is rather similar to the behavior of link costs. By capacity increment, since the number of located facilities decrease, demands must be carried to the facilities to be served, and therefore transportation cost increase. From Fig. 14 we see the objective appears approximately piecewise-linear convex in link capacity. We henceforth indicate the capacity after which further capacity increases do not decrease the objective (i.e., after which the problem is essentially uncapacitated), as $U_{\max }$. From the figure, we see that $U_{\text {max }}$ is about 70 units.

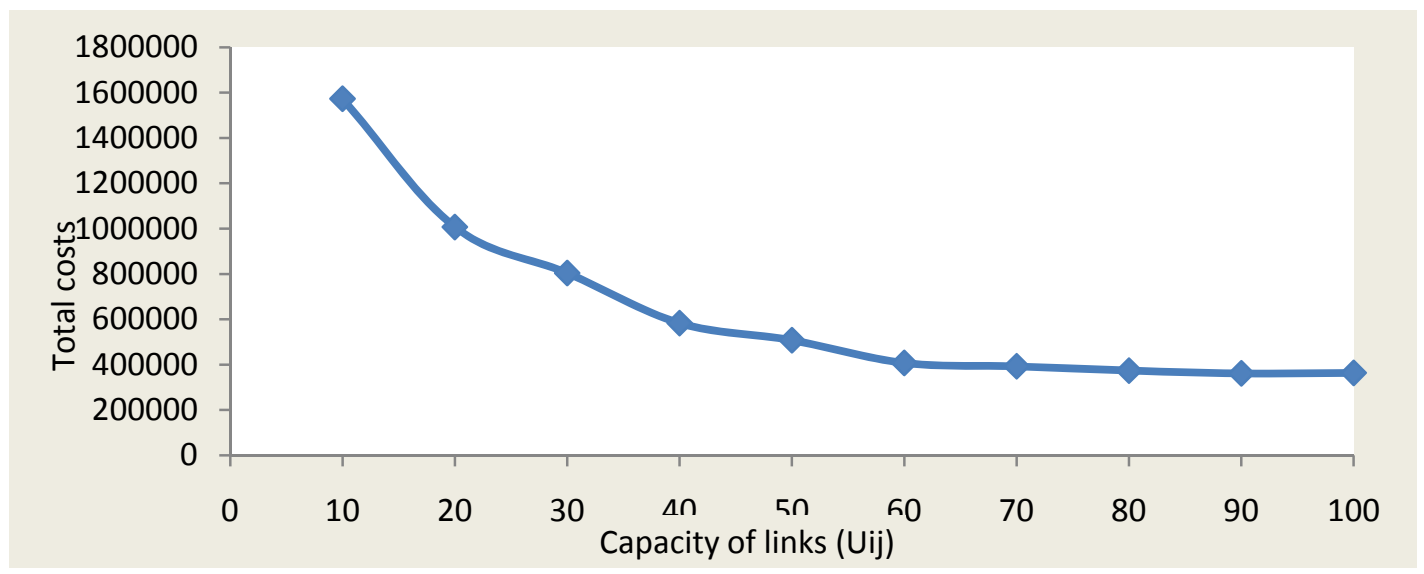

Fig. 14. Total costs vs. capacity

\section{Solution procedure for bi-objective problem}

Since we present a bi-objective model for the problem, we should use one of the multi attribute technique for solving the problem. Generally, in multi attribute optimizations, we intend to select the best alternative concerning the given criteria. While in most cases, criteria are in conflict with each others, and usually an improvement in one objective could deteriorate in other objectives, finding a unique solution which optimizes all objectives is impossible. Therefore a set of solutions is provided for the decision maker to choose a desirable solution. We call this set of solutions as non-dominated solutions or Pareto set. The motivation of this denomination is that there is no feasible solution of the problem which can dominate any solutions of the non-dominated set.

Pareto chart is one of the important tools in multi criteria decision making. One of the simplest ways to draw Pareto chart is to put one of the objective function as a constraint. In this paper for drawing Pareto chart we use this technique, the second objective function $\left(f_{2}\right)$ is considered as a constraint and a single objective problem is obtained as follow,

$$
\left\{\begin{array} { l } 
{ \operatorname { M i n } \sum _ { ( i , j ) \epsilon L } \sum _ { k \epsilon N } t _ { i j } ^ { k } Y _ { i j } ^ { k } } \\
{ \text { Min } \sum _ { i } f _ { i } Z _ { i } + \sum _ { ( i , j ) } C _ { i j } X _ { i j } } \\
{ \quad \text { Subject to } } \\
{ \quad ( 3 ) - ( 1 1 ) }
\end{array} \quad \Rightarrow \left\{\begin{array}{c}
\operatorname{Min} \sum_{(i, j) \epsilon L} \sum_{k \epsilon N} t_{i j}^{k} Y_{i j}^{k} \\
\text { Subject to } \\
\sum_{i} f_{i} Z_{i}+\sum_{(i, j)} C_{i j} X_{i j} \leq \Gamma \\
(3)-(11)
\end{array}\right.\right.
$$

First we calculate the minimum and maximum value for the second objective function. To calculate the minimum value, we solve the problem just with the second objective function and for calculating the maximum value, we solve the problem only with the first objective function and then put the optimal decision vector in the second objective function and calculate the value for this solution.

Now by changing the value of $\Gamma$ between minimum and maximum value, we solve the problem and find the objective value for the given $\Gamma$ and draw the Pareto chart using these points.

The accuracy of Pareto chart will increase by using more numbers of $\Gamma$ for drawing Pareto chart. The Pareto chart of the 21-node problem is shown in Fig. 15. using 37 different values for $\Gamma$. 


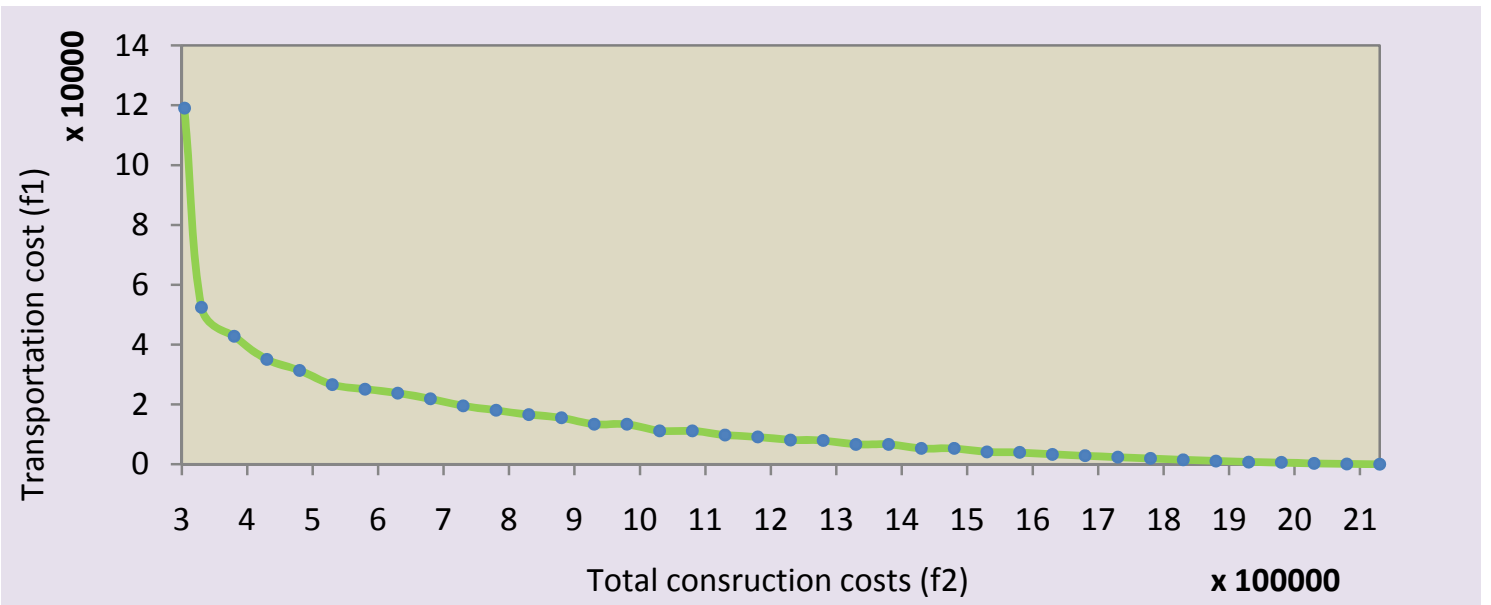

Fig. 15. Pareto chart for the 21-nodes instance

\section{Conclusions}

In this paper we have introduced an extension of the CFLNDP in which the maximum amount of demands can be carried by a link is limited. This problem has a number of important applications in regional planning, distribution, telecommunications, energy management, and other areas.

We presented a bi-objective mixed integer programming formulation of the problem and extended a hybrid algorithm to solve the resulted problem. The proposed hybrid algorithm has been tested on 12 problem instances and the results have been compared with those of obtained by the exact solution procedure. Numerical results indicate that the proposed method of this paper provides solutions with relatively good quality. The resulted multi-objective problem is solved using Lexicography method for a well-known example from the literature with 21 nodes. Finally, sensitivity analysis on a test problem was conducted to gain insight into the model's behaviour. Compared to the uncapacitated model, when capacity constraints are imposed, we observed that the problem become more difficult to solve and link costs actually increase.

\section{References}

Balakrishnan, A., Magnani, T.L., \& Wong, R.T. (1989). A dual-ascent procedure for large-scale uncapacitated network design. Operation Research, 37(5), 716-740.

Bazaraa, M. S., Jarvis, J. J., \& Sherali, H. D. (1990). Linear programming and network flows. New York: Springer.

Berman, O., Ingco, D.I., Odoni, A.R., (1992). Improving the location of minisum facilities trough network modification. Annals of Operations Research 40(1), 1-16.

Bhadury, J., Gewali, L.P., Chandrasekaran, R., \& Badr, M., (2003). Network design subject to facility location. 15th Canadian Conference on Computational Geometry.

Campbell, J.F., (1994). A survey of network hub location. Studies in Locational Analysis 6, 31-49.

Daskin, M.S., \& Jones, P.C. (1993). A new approach to solving applied location/allocation Problems. Computer-aided Civil and Infrastructure Engineering, 8(6), 409-421.

Daskin, M.S., Snyder, L.V., \& Berger, R.Y.(2005). Facility location in supply chain design. Logistics Systems: Design and Optimization. 39-65.

Drezner, Z., Wesolowsky, G. O. (1998). Design-location problems. INFORMS National Meeting, seattle.

Drezner, Z., \& Wesolowsky, G. O. (2003). Network design: selection and design of links and facility location. Transportation Research Part A. 37(3), 241-256.

Friesz, T.L., Shah, S., \& Bernstein, D. (1998). Disequilibrium network design: a new paradigm for transportation planning and control. In: Network Infrastructure and the Urban Environment: Advances in Spatial Systems Modeling. Springer, Berlin. 
Klincewicz, J.G. (1998). Hub location in backbone/tributary network design: a review. Location Science, 6(1-4), 307-335.

Kirkpatrick S, Gelatt jr. CD, \& Vecchi MP. (1983). Optimization by simulated annealing. Science. 220, 671-680.

Laporte, G., (1998). Location-routing problems. In Golden, B.L., Assad, A.A.(Eds), Vehicle Routing: Methods and studies. North-Holland, Amsterdam, 163-198.

Magnanti, T.L. \& Wong, R.T. (1984). Network design and transportation planning: models and algorithms. Transportation Science, 18(1), 1-55.

Melkote, S., (1996). Integrated models of facility location and network design. PhD Dissertation. Department of Industrial Engineering and Management Sciences, Northwestern University, Evanston, IL.

Melkote, S., \& Daskin, M. S. (2001). An integrated model of facility location and transportation network design. Transportation Research Part A: Policy and Practice, 35(6), 515-538.

Melkote, S., \& Daskin, M. S. (2001). Capacitated facility location-network design problems. European Journal of Operational Research, 129(3), 481-495.

Metropolis, N., Rosenbluth, A.W, Rosenbluth, M.N., Teller, A.H, \& Teller, E. (1953). Equations of state calculations by fast computing machines. Journal of chemical- physics, 21(6). 1087-1092.

Mitchel, D.H., \& Smith, J.M. (2001). Topological network design of pedestrian networks. Transportation Research Part B: Methodological, 35(2), 107-135.

Peeters, D., \& Thomas,I. (1995). The effect of spatial structure on p-median results. Transportation Science, 29(4), 366-373.

Pardalos, P.M., \& Du, D. (1998). Network design: Connectivity and facilities location. In: DIMACS Series in Discrete Mathematics and Theoretical Computer Science, 40. American Mathematical Society.

Ravi, R., \& Sinha, A. (2006). Approximation algorithms for problems combining facility location and network design. Operations Research. 54. 73-81

Solanki, R.S., Gorti, J.K., \& Southworth, F. (1998). Using decomposition in large-scale highway network design with a quasi-optimization heuristic. Transportation Research Part B: Methodological ,32(2), 127-140.

Yang, H. (1998). Models and algorithms for road network design: a review and some new developments. Transport Reviews 18(3), 257-278. 